\title{
Ectopic Expression of PA2.26 Antigen in Epidermal Keratinocytes Leads to Destabilization of Adherens Junctions and Malignant Progression
}

\author{
Francisco G. Scholl, Carlos Gamallo, and Miguel Quintanilla \\ Instituto de Investigaciones Biomédicas Alberto Sols CSIC-UAM (FGS, MQ) and Departamento de Anatomía Patológica \\ (CG), Hospital Universitario de la Princesa, Facultad de Medicina, Universidad Autónoma de Madrid, Madrid, Spain
}

\begin{abstract}
SUMMARY: PA2.26 antigen is a small mucin-type transmembrane glycoprotein induced in mouse epidermal keratinocytes during carcinogenesis. It is located at plasma membrane projections, such as microvilli and ruffles, where it interacts with the actin cytoskeleton. Previous studies revealed that ectopic expression of PA2.26 in epidermal MCA3D keratinocytes induces cell surface extensions and increased motility. Here, we show that PA2.26-expressing MCA3D (3D2.26) cell transfectants undergo a phenotypic conversion linked to the acquisition of malignant characteristics. The 3D2.26 cells down-regulate basal keratin K14 and up-regulate vimentin and keratin K8 expression. Immunofluorescence analysis in 3D2.26 cell cultures showed loss of cortical actin filaments and destabilization of adherens junctions mediated by E- and P-cadherin, although both cadherin mRNAs were expressed in the transfectants. When the cadherin protein levels were analyzed in Western blots, no P-cadherin protein or smaller polypeptide E-cadherin forms were detected, suggesting that E- and P-cadherin synthesized in 3D2.26 cells was unstable and proteolytically degraded. Transplantation of 3D2.26 cells into athymic nude mice induced tumors, whereas MCA3D cells and control (3DN) transfectants were not tumorigenic after 72 days postinjection. The phenotype of the tumors was undifferentiated, with mixed regions exhibiting a glandular differentiation pattern in which the presence of numerous surface microvilli was observed at the ultrastructural level. Interestingly, PA2.26 antigen was highly expressed in these microvillous cell surfaces. Tumor cells were vimentin- and K8-positive and showed an aberrant pattern of E-cadherin protein expression in which large cytoplasmic aggregates were found close to the nucleus. Infiltration of tumor cells into lymphatic vessels and the presence of frequent regional lymph node metastases were also observed in the tumors. These results indicate that expression of PA2.26 antigen in premalignant keratinocytes induces a fully transformed and metastatic phenotype, and they suggest an involvement of PA2.26 in malignant progression. (Lab Invest 2000, 80:1749-1759).
\end{abstract}

$P^{\prime \prime 2 r a t}$ A2.26 antigen was identified as a cell-surface protein of about $45 \mathrm{kDa}$ induced in basal-like keratinocytes and dermal fibroblasts during mouse epidermal carcinogenesis and skin remodeling processes. PA2.26 is absent from cultured nontumorigenic keratinocytes, but it is expressed in carcinoma cell lines and cultured active fibroblasts (Gandarillas et al, 1997). The molecular and biochemical characterization of PA2.26 revealed that it is a small mucin-like transmembrane sialoglycoprotein of 172 amino acids, located at microvilli, filopodia, lamellipodia, and ruffles. In normal tissues, PA2.26 is expressed in different type of cells: in the epithelia of choroid plexuses, ependyma, glomeruli, and alveoli, in all mesothelia, and in endothelia of lymphatic vessels, always con-

Received August 10, 2000.

This work was supported by Grants SAF98-0085-CO3-02 (to MQ) and -03 (to CG) from the "Comisión Interministerial de Ciencia y Tecnología" (CICYT), and 08.1/0021.1/99 from the "Comunidad Autónoma de Madrid" (CAM). FGS was the recipient of a postdoctoral fellowship from the Comunidad Autónoma de Madrid.

Address reprint requests to: Dr. Miguel Quintanilla, Instituto de Investigaciones Biomédicas Alberto Sols CSIC-UAM, Arturo Duperier 4, 28029Madrid, Spain. Fax: 3491 5854587; E-mail:mquintanilla@iib.uam.es centrated at the apical plasma membranes and microvillous projections (Scholl et al, 1999).

OTS-8 and gp38, two previously described mouse proteins, exhibit amino acid sequences highly homologous to that of PA2.26 and are likely encoded by the same gene (Scholl et al, 1999). OTS-8 was isolated as a phorbol ester-inducible gene from cultured osteoblasts (Nose et al, 1990), and gp38 was described as a marker of stromal cells in T-cell-dependent areas of peripheral lymphoid tissues (Farr et al, 1992). The rat (T1 $\alpha /$ E11/podoplanin), canine (gp40), and human (gp36) homologs to murine PA2.26 have also been reported by different laboratories (Breiteneder-Geleff et al, 1997; Rishi et al, 1995; Wetterwald et al, 1996; Zimmer et al, 1997, 1999). All these proteins have similar biochemical characteristics and tissular distributions. Thus, for example, rat $\mathrm{T} 1 \alpha$ sialoglycoprotein was first described as a marker of alveolar type I epithelial cells of the lung (Rishi et al, 1995), and human gp36 has been recently characterized as a mucin-type transmembrane sialoglycoprotein expressed at the apical plasma membrane of the vascular endothelium and alveolar epithelium (Zimmer et al, 1999). Although canine gp40 can act as a main receptor for the influenza C virus (Zimmer et al, 1995), and rat podoplanin was found to be down-regulated in 
an experimental model of minimal-change nephropathy (Breiteneder-Geleff et al, 1997), the biological functions of these proteins are unknown.

We found that PA2.26 antigen co-localizes and co-immunoprecipitates with proteins of the ezrin, radixin, moesin (ERM) family (Scholl et al, 1999). ERM proteins, which are related to the band 4.1 superfamily and the tumor suppressor, neurofibromatosis type 2 (NF2) (merlin/schwannomin), are concentrated in cellsurface projections, where they link the actin cytoskeleton to plasma membrane proteins. They play structural and regulatory roles in the formation and maintenance of those dynamic plasma membrane structures and are involved in cell motility (Mangeat et al, 1999). PA2.26 is thought to physically interact with the actin cytoskeleton via its association with ezrin and moesin (but not with radixin) through a cluster of basic amino acids present in the cytoplasmic domain of the PA2.26 molecule. Ectopic expression of PA2.26 in keratinocytes induces plasma membrane extensions, concomitantly with a major reorganization of the actin cytoskeleton and relocalization of ezrin to cellsurface projections. These changes are associated with an epithelial-fibroblastoid morphological conversion and increased motility (Scholl et al, 1999).

In this article, we show that expression of PA2.26 in premalignant keratinocytes (MCA3D cell line) leads to destabilization of adherens junctions mediated by $\mathrm{E}-$ and P-cadherin, down-regulation of basal keratins, induction of vimentin and keratin $\mathrm{K} 8$, and the acquisition of a malignant phenotype.

\section{Results}

\section{Cloning of PA2.26-Expressing MCA3D Keratinocyte Cells and Characterization of Differentiation Markers}

MCA3D keratinocytes were transfected with the mouse PA2.26 cDNA cloned into the pcDNA3 vector. Stable clones were isolated after selection in G418 (designated as 3D2.26). Control clones transfected with the plasmid vector alone were also obtained (designated as 3DN). All 10 MCA3D-derived 3D2.26 clones showed a similar change to a fibroblastoid morphology and were motile in a wound-healing assay, whereas 3DN clones retained the epithelial morphology and static characteristics of MCA3D cells (Scholl et al, 1999). (See Fig. 1, A and B, for morphological appearances of 3DN and 3D2.26 cells, respectively.)

To study whether this morphological conversion was associated with changes in differentiation-specific markers, we analyzed the expression of epithelial (keratins, cadherins) and mesenchymal (vimentin) genes either by Northern hybridization experiments or by reverse transcriptase-polymerase chain reaction (RT-PCR). As
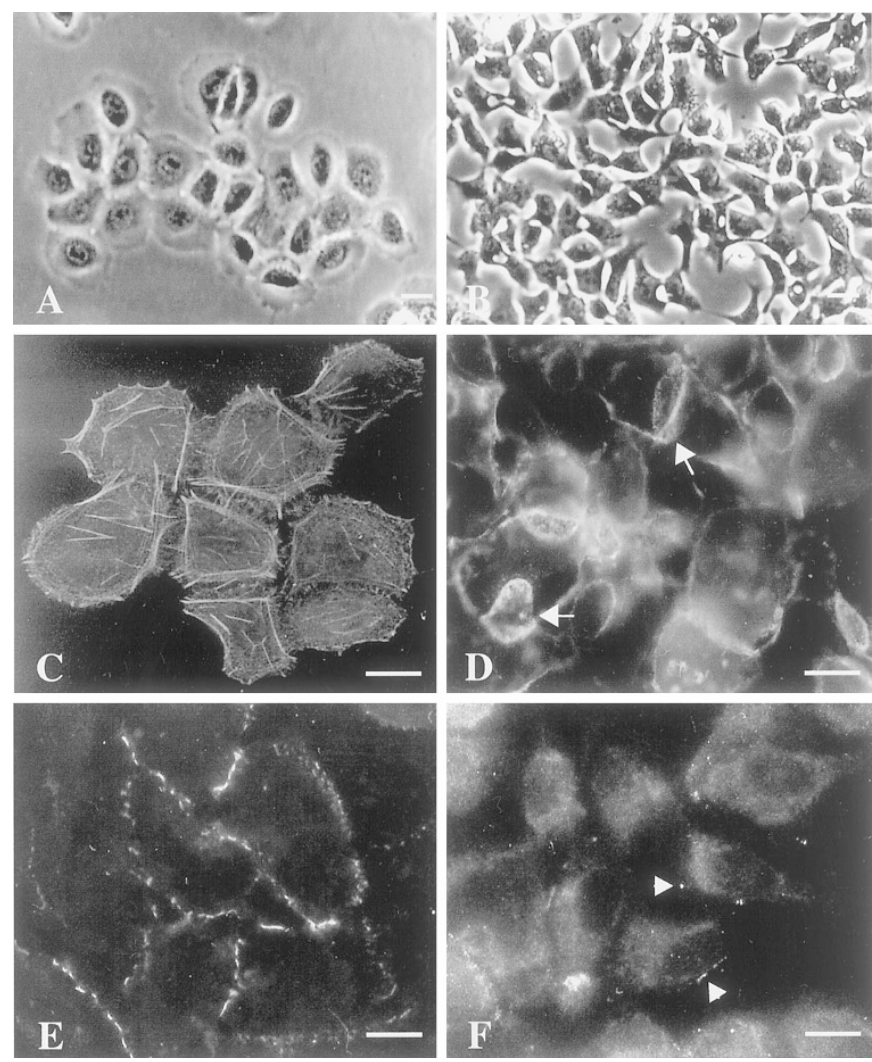

Figure 1.

$A$ and $B$, Morphological appearances of control 3DN5 $(A)$ and PA2.26-expressing 3D2.26-7 (B) cells. $C$ and $D$, Immunofluorescence analysis of F-actin in 3DN5 (C) and 3D2.26-13 (D) cells. Arrows mark ruffles formed between two adjacent cells. $E$ and $F$, Immunofluorescence analysis of E-cadherin in 3DN5 (E) and 3D2.26-13 $(F)$ cells. Arrowheads point to small aggregates at the cell surface. The figure shows representative images of the indicated clones. All other clones examined (3DN9, 3D2.26-1, 3D2.26-4, 3D2.26-6, and 3D2.26-11) exhibited similar characteristics. 
shown in Figure 2, 3D2.26 clones switched off the expression of the basal keratin $\mathrm{K} 14$ and induced keratin K8 (Fig. 2A, lanes 3-7) and vimentin (Fig. 2B, lanes 3-5) messages. Coexpression of the embryonic/simple epithelial keratin $\mathrm{K} 8$ and vimentin is characteristic of transformed keratinocytes, as can be seen in PDV and HaCa4, two PA2.26-expressing squamous carcinoma cell lines (Gandarillas et al, 1997) used in this experiment as additional controls (Fig. 2A, lanes 8-9; Fig. 2B, lanes $6-7)$.

We also analyzed by RT-PCR the expression of Eand $\mathrm{P}$-cadherin cell-cell adhesion molecules typical of epidermal keratinocytes (Navarro et al, 1991). The 3D2.26 clones maintained the expression of E- and $\mathrm{P}$-cadherin messages, although a slight reduction in the levels of E-cadherin mRNA was observed in PA2.26-expressing transfectants (Fig. 3A, lanes 3-5) compared with the parental cell line or 3DN5 control cells (Fig. 3A, lanes 1-2). Notwithstanding this, the intact mature cadherin proteins of molecular masses

A

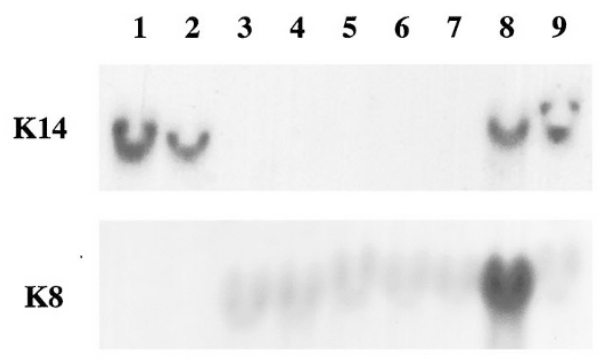

$7 \mathrm{~S}$

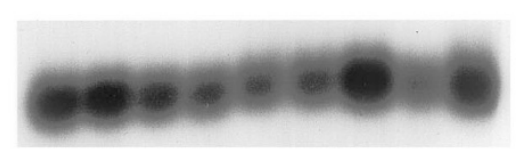

B

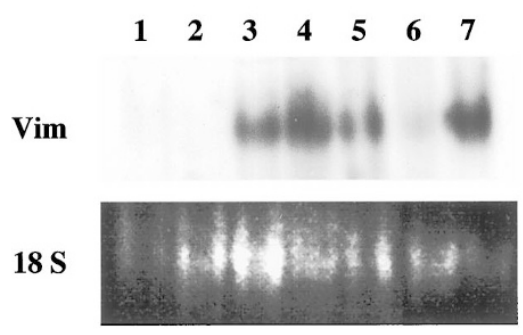

Figure 2.

Expression of intermediate filament proteins mRNAs in cell lines. A, Poly $(\mathrm{A})^{+}$-enriched RNA $(20 \mu \mathrm{g})$ from MCA3D- (lane 1), 3DN5- (lane 2), PA2.26expressing transfectants: 3D2.26-1 (lane 3), 3D2.26-4 (lane 4), 3D2.26-10 (lane 5), 3D2.26-11 (lane 6), 3D2.26-13 (lane 7), and carcinoma cell lines, PDV (lane 8) and HaCa4 (lane 9), were subjected to Northern blot analysis with K14 and K8 probes. The bottom panel shows hybridization with a 7S RNA probe to normalize the loading. $B$, Northern blot analysis of MCA3D (lane 1), 3DN5 (lane 2), 3D2.26-4 (lane 3), 3D2.26-11 (lane 4), 3D2.26-13 (lane 5), PDV (lane 6), and $\mathrm{HaCa} 4$ (lane 7 ) poly $(\mathrm{A})^{+}$-enriched RNAs with a vimentin probe. The bottom panel shows the ethidium bromide stain of the filter corresponding to 18S RNA. of $124 \mathrm{kDa}$ (E-cadherin) and $119 \mathrm{kDa}$ (P-cadherin) could not be detected in the 3D2.26 cell lysates by Western immunoblotting using the monoclonal antibodies (mAbs) ECDD-2 and PCD-1, specific for E- and $\mathrm{P}$-cadherin, respectively (Figure 3B, lanes 3-5). Furthermore, several bands of sizes lower than $124 \mathrm{kDa}$ $(115,90,58-60 \mathrm{kDa})$ were recognized in the blots by the mAb ECCD-2, whereas no polypeptide bands were detected with the PCD-1 mAb. These results suggested that cadherin proteins synthesized in 3D2.26 cells were unstable and proteolytically degraded. On the other hand, the levels of $\beta$-catenin, a cytoplasmic protein that links cadherin receptors to the actin cytoskeleton through its interaction with $\alpha$-catenin in adherens junctions (Ozawa and Kemler, 1992), were unchanged in the transfectants (Fig. 3B), and $\beta$-catenin served as an internal protein loading marker.

PCD-1 anti-P- and ECCD-2 anti-E-cadherin immunofluorescence analysis in MCA3D and control 3DN5 cells revealed the typical keratinocyte pattern of cadherin staining as a continuous line of dots at cell-cell contacts (Navarro et al, 1991) (Fig. 1E). In 3D2.26 transfectants, however, the overall E-cadherin fluorescence intensity was drastically reduced, and the protein was found in small aggregates, either at the plasma membrane or in the cytoplasm (Fig. 1F). In the case of P-cadherin, immunofluorescence staining in 3D2.26 cells was completely negative (not shown). These changes in E- and $\mathrm{P}$-cadherin protein expression/organization occurred concomitantly with a major rearrangement of the actin cytoskeleton. Thus, 3DN cells showed strong cortical actin bundles and stress fibers, characteristics of keratinocytes (Fig. 1C), which were lost in 3D2.26 cells. Actin filaments in the latter cells appeared to concentrate at the cell periphery in plasma membrane projections (Fig. 1D, arrows). (See Scholl et al, 1999, for a more detailed description of actin cytoskeletal reorganization in 3D2.26 transfectants.)

We also analyzed the expression of keratins and vimentin by immunofluorescence analysis and found that 3DN cells were K14-positive and K8- and vimentin-negative, as previously reported for MCA3D cells (Caulín et al, 1993a, 1993b) and in accordance with the Northern blot data (Fig. 2). The 3D2.26 cells, on the other hand, were K14-negative and K8- and vimentin-positive (data not shown; see paragraph below on immunohistological staining in 3D2.26-induced tumors).

\section{Tumorigenic and Metastatic Properties of 3D2.26 Cell Transfectants}

The tumorigenic properties of the cell transfectants were examined by transplantation into athymic nude mice. In a first experiment, the parental cell line, two control clones (3DN5 and 3DN9), and three PA2.26transfected clones (3D2.26-4, -11, and -13) were injected into the two flanks of the animals (Table 1, Experiment 1). All 3D2.26 clones produced tumors that grew to sizes close to or highly above $1 \mathrm{~cm}^{2}$ at the end of the experiment (72 days postinjection), 
$\mathbf{A}$

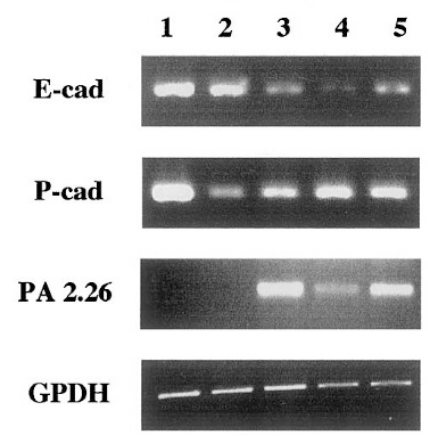

B

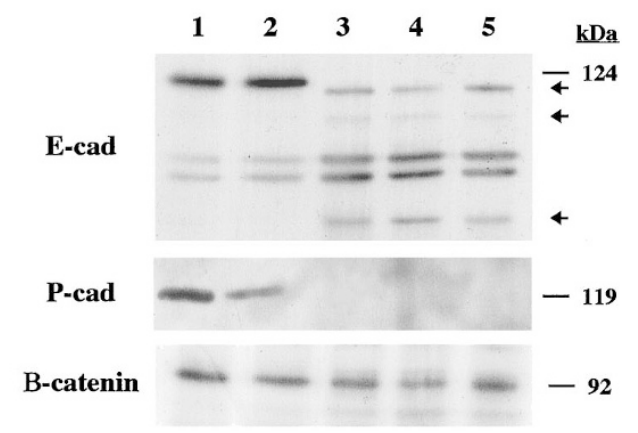

Figure 3.

Analysis of E-cadherin, P-cadherin, and $\beta$-catenin expression in MCA3D cells and derived transfectants. $A$, Poly $(A)^{+}$RNA from MCA3D (lane 1), 3DN5 (lane 2), 3D2.26-4 (lane 3), 3D2.26-11 (lane 4), and 3D2.26-13 (lane 5) were subjected to RT-PCR with E-cadherin, P-cadherin, PA2.26, and glyceraldehyde-3-phosphate dehydrogenase (GPDH) oligonucleotides. B, Detection of E-cadherin, P-cadherin, and $\beta$-catenin protein levels by Western blot analysis with mAbs ECCD-2, PCD-1, and $\mathrm{C} 14$, respectively. Lanes are as in panel $A$. Molecular masses of intact proteins are indicated in kDa. Arrows mark E-cadherin polypeptide fragments.

Table 1. Tumorigenic Characteristics of MCA3D Transfectant Cell Clones ${ }^{a}$

\begin{tabular}{|c|c|c|c|c|c|}
\hline \multirow[b]{2}{*}{ Experiment } & \multirow[b]{2}{*}{ Cell line } & \multirow{2}{*}{$\begin{array}{c}\text { Tumor } \\
\text { incidence }{ }^{b}\end{array}$} & \multicolumn{2}{|c|}{$\begin{array}{l}\text { Number of tumors with } \\
\text { sizes (in } \mathrm{cm}^{2} \text { ): }\end{array}$} & \multirow{2}{*}{$\begin{array}{l}\text { Lymph } \\
\text { node } \\
\text { metastasis }^{c}\end{array}$} \\
\hline & & & $0.5-1.0$ & $>1.0$ & \\
\hline \multirow{6}{*}{1} & MCA3D & $0 / 4$ & - & - & - \\
\hline & 3DN5 & $0 / 6$ & - & - & - \\
\hline & 3DN9 & $0 / 6$ & - & - & - \\
\hline & 3D2.26-4 & $6 / 6^{d}$ & 3 & 3 & $(+)^{d}$ \\
\hline & 3D2.26-11 & $4 / 6$ & 4 & 0 & ND \\
\hline & $3 \mathrm{D} 2.26-13$ & $6 / 6$ & 3 & 3 & ND \\
\hline \multirow{3}{*}{2} & 3DN5 & $0 / 2$ & - & - & - \\
\hline & 3D2.26-4 & $4 / 4^{e}$ & 2 & 2 & $1 / 4$ \\
\hline & $3 \mathrm{D} 2.26-11$ & $4 / 4$ & 1 & 3 & $3 / 4$ \\
\hline
\end{tabular}

\footnotetext{
${ }^{a}$ Groups of Swiss athymic nude mice were inoculated id with $3 \times 10^{6}$ cells in the two flanks (Experiment 1 ) or at a single site on the back close to the neck (Experiment 2).

${ }^{b}$ The tumor incidence at 72 days postinjection (Experiment 1 ) and 64 days postinjection (Experiment 2 ) is expressed as the number of tumors developed per total number of injection sites.

${ }^{c}$ The number of tumors that produced lymph node metastases was recorded only in the Experiment 2. Infiltration of tumor cells into the lymph nodes was confirmed by histological analysis. ND, not determined.

${ }^{d}$ A mouse bearing two tumors of $0.7 \mathrm{~cm}^{2}$ and $2.4 \mathrm{~cm}^{2}$ died at Day 49 postinjection. In this animal the presence of a lymph node metastasis was observed after histology, although the incidence of metastasis was not recorded.

${ }^{e} \mathrm{~A}$ mouse bearing a tumor of $0.5 \mathrm{~cm}^{2}$ died at Day 47 postinjection. Autopsy revealed an intestinal occlusion as the probable cause of death.
}

whereas the parental cell line and control clones were not tumorigenic. Clone 3D2.26-4 exhibited the most aggressive behavior, killing an animal bearing two tumors (2.4 $\mathrm{cm}^{2}$ [right flank] and $0.7 \mathrm{~cm}^{2}$ [left flank]) at 49 days postinjection. Examination of the right flank of this mouse revealed a tumoral mass and a satellite node independent of the tumor (Fig. 4A); the latter was shown to contain a lymph node with metastasis after histology (Fig. 4B). The histological analysis of the tumors revealed solid nests of cells with large eosinophilic cytoplasms and nuclei with conspicuous nucleoli. In some areas, tumor cells presented a glandular differentiation pattern (Fig. 4C, arrowheads) with periodic acid Schiff (PAS)-positive material accumulated (not shown). In these regions, the surfaces of the cavities that formed between two adjacent cells were intensely stained with the PA2.26 mAb (Fig. 4D). Electron microscopy analysis revealed that the apical domains of both cells in the cavities contained microvillous projections facing the lumen (Fig. 4E, arrowheads). These results are in agreement with our previous finding that PA2.26 antigen is a component of plasma membrane microvilli in cultured cells and tissues (Scholl et al, 1999).

To study in more detail the metastatic behavior of 3D2.26 clones, we performed a second experiment in which cells were injected at a single site on the back close to the neck of each animal. Besides dissecting primary tumors, our interest in this experiment was focused on finding metastasis in regional lymph nodes. The 3D2.26-4 and -11 clones produced tumors with incidences of $100 \%$ at 64 days postinjection. Control 3DN5 cells, on the other hand, were not tumorigenic (Table 1, Experiment 2). As shown in Figure 5, the histological patterns of the tumors were similar to those of the first experiment with glandular 

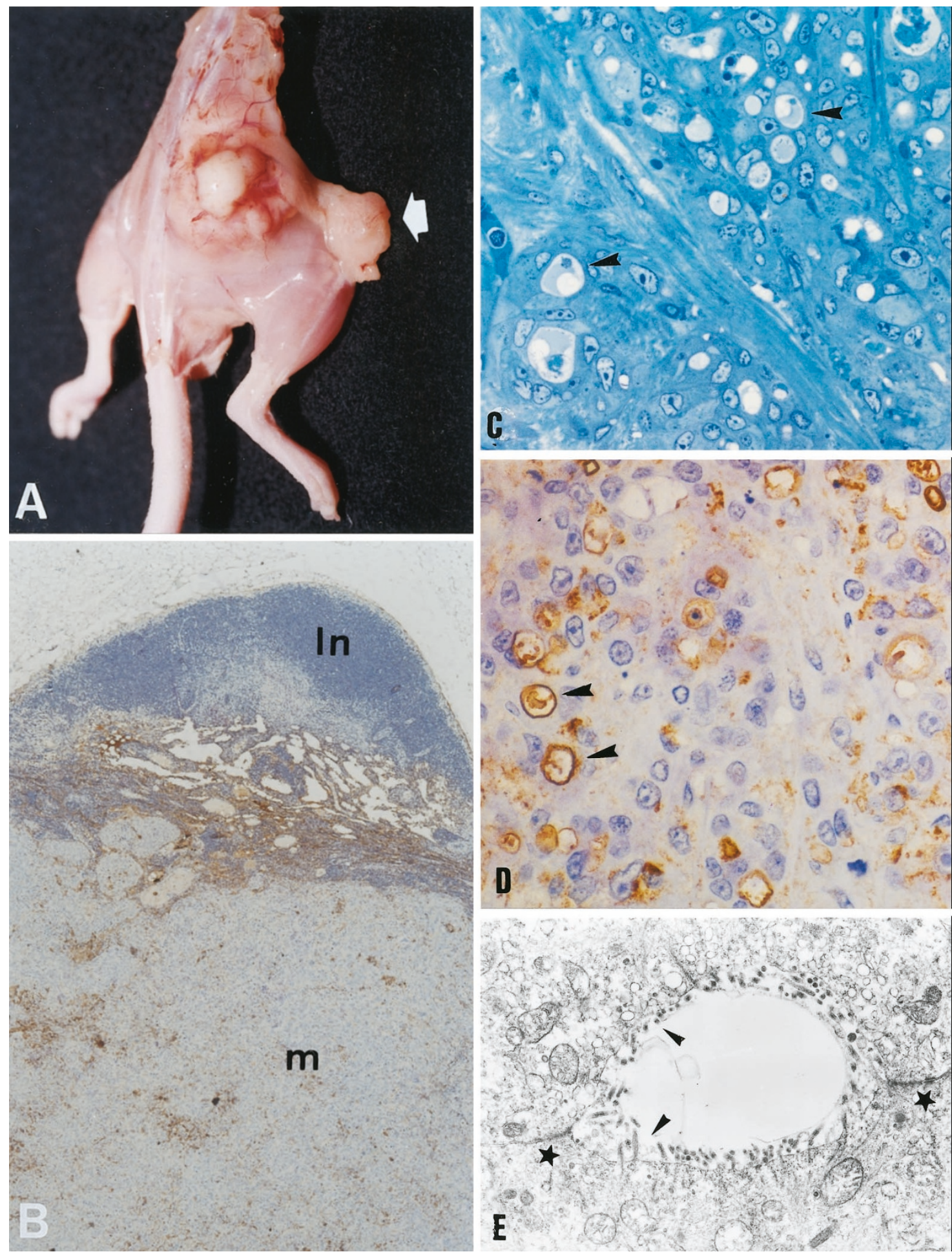

\section{Figure 4.}

A, Tumor on the flank of a mouse injected with 3D2.26-4 cells (corresponding to the Experiment 1 of Table 1) showing a satellite node (arrow). B, Lymph node (In) metastasis $(\mathrm{m})$ corresponding to the satellite node showed in panel $A$, stained with anti-PA2.26 mAb. $C$ to $E$, Immunohistological and ultrastructural analysis of 3D2.26-induced tumors. C, Semi-thin section showing a glandular differentiation pattern. Arrowheads mark cavities with mucinous material accumulated. D, PA2.26 immunohistological staining in areas of glandular differentiation. Note that PA2.26 antigen is concentrated at the apical surface of cells forming the cavities (arrowheads). E, Electron microscopy showing numerous plasma membrane microvilli facing the lumen in the cavities (arrowheads). Asterisks indicate cell-cell boundaries. Sections in panels $C$ to $E$ correspond to the tumor presented in panel $A$.

differentiation (Fig. 5A) mixed with poorly differentiated areas (Fig. 5B). Infiltration of tumor cells into lymphatic vessels was a common finding in the ma- jority of the tumors (Fig. 5C). In the macroscopic examination of tumors, seven lymphadenopathies were detected in four animals (Table 1, Experiment 2) 


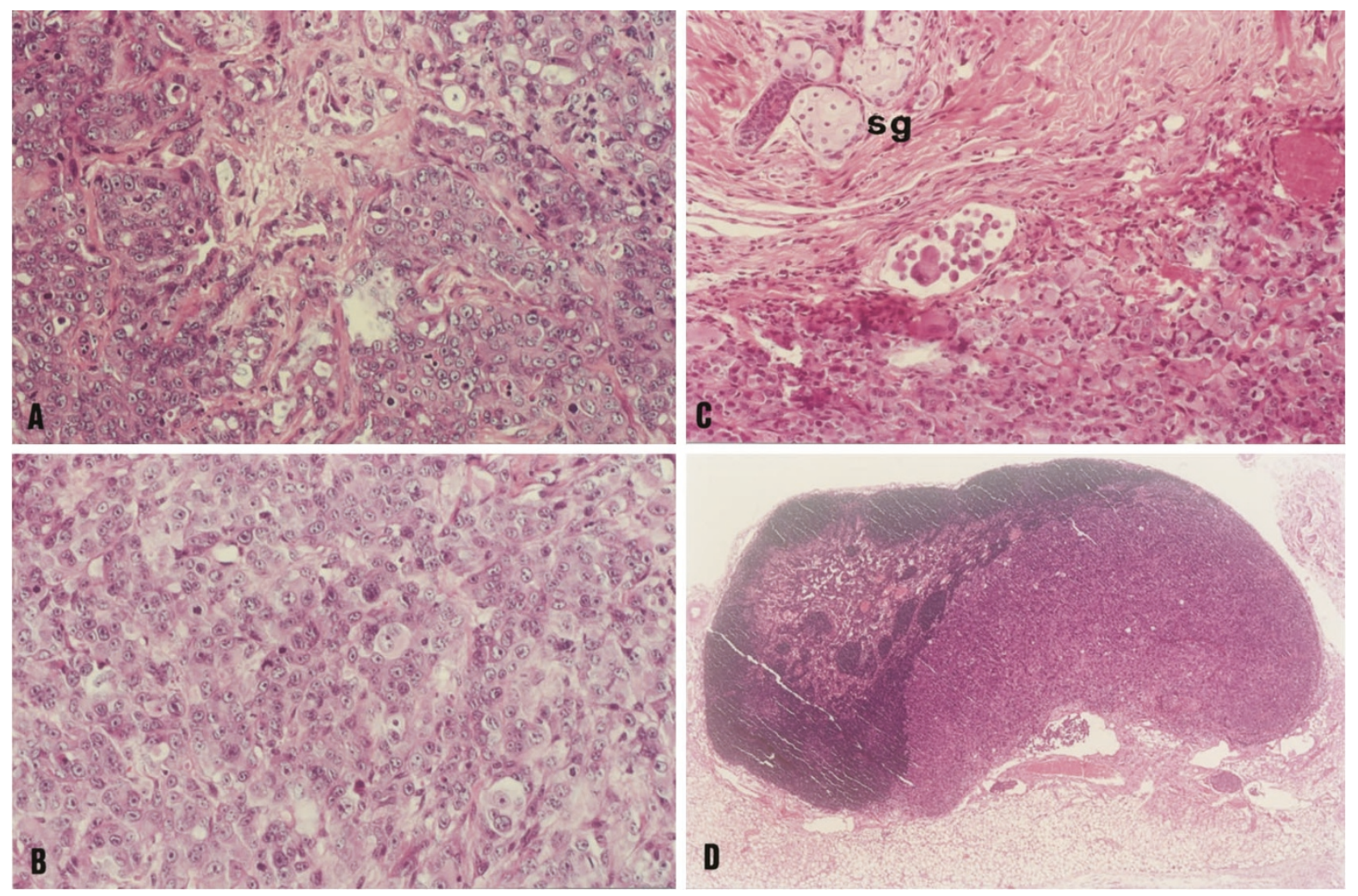

Figure 5.

Histological appearance of tumors induced by 3D2.26-11 cells in nude mice (corresponding to Experiment 2 of Table 1). $A$ and $B$, Sections showing a glandular differentiation component $(A)$ and a poorly differentiated region $(B)$ of the same tumor. $C$, Infiltration of tumor cells into a lymphatic vessel. Note the presence of sebaceous glands $(\mathrm{sg})$ in the skin in the upper left corner. $D$, Lymph node metastasis.

in which metastasis was assessed by histological analysis (Fig. 5D).

Immunohistological staining of tumor sections showed that anti-PA2.26 staining was intense in areas of glandular differentiation (Fig. 4D), but decreased in undifferentiated areas of tumors and metastases (Fig. 4B). In the poorly differentiated regions, many tumor cells were PA2.26-negative, whereas others showed the antigen located at the plasma membrane either surrounding the entire cell surface or circumscribed to some zones of the cell surface (Fig. 6A). Anti-vimentin and anti-K8 staining in tumor sections were both positive and homogeneous (Fig. 6, C and D), indicating that both intermediate filament proteins are coexpressed together within the same cells. Interestingly, K8 appeared surrounding the lumen in areas of glandular differentiation (arrowheads in Fig. 6C). No staining for $\mathrm{P}$-cadherin was observed with the anti-Pcadherin mAb C56 (not shown), whereas anti-Ecadherin mAb C36 revealed an anomalous pattern of E-cadherin distribution in tumor cells, with no staining or weak staining at cell-cell boundaries and the presence of large juxta-nuclear cytoplasmic aggregates (Fig. 6B, arrowheads).

\section{Discussion}

PA2.26 antigen was identified in our laboratory as a cell-surface protein induced in basal-like epidermal ker- atinocytes during wound healing and chemical carcinogenesis (Gandarillas et al, 1997). To analyze the biological function of PA2.26 in epidermal cells, we transfected the mouse PA2.26 cDNA in MCA3D keratinocytes, which do not express the antigen. MCA3D cells were derived from primary epidermal cultures treated with the carcinogen 7,12-dimethylbenz(a)anthracene and selected on the basis of their resistance to $\mathrm{Ca}^{2+}$-induced terminal differentiation (Kulesz-Martin et al, 1983). MCA3D keratinocytes have normal Ras, p53, p15 ${ }^{\mathrm{INK} 4 \mathrm{~B}}$, and $\mathrm{p} 16^{\mathrm{INK} 4 \mathrm{~A}}$ genes and are nontumorigenic upon injection in nude mice (Burns et al, 1991; Linardopoulos et al, 1995; Quintanilla et al, 1991). However, they have a tetraploid content of DNA and have been found to spontaneously develop tumors in skin graft experiments (Kulesz-Martin et al, 1983; Strickland et al, 1988). Therefore, MCA3D can be considered a premalignant cell line. In this work, we demonstrate that ectopic expression of PA2.26 antigen in MCA3D keratinocytes induces an epithelial-fibroblastoid phenotypic conversion associated with the acquisition of malignant characteristics. Phenotypic changes include down-regulation of basal keratins (K14), up-regulation of vimentin and K8 (Fig. 2), and loss of adherens junctions mediated by $\mathrm{E}$ - and P-cadherin (Figs. 1 and 3). 3D2.26 clones express the exogenous PA2.26 mRNA of $1.2 \mathrm{~Kb}$, and induction of the endogenous message (1.9 Kb) was not observed in the transfectants (Scholl et al, 1999), ruling out spontaneous 

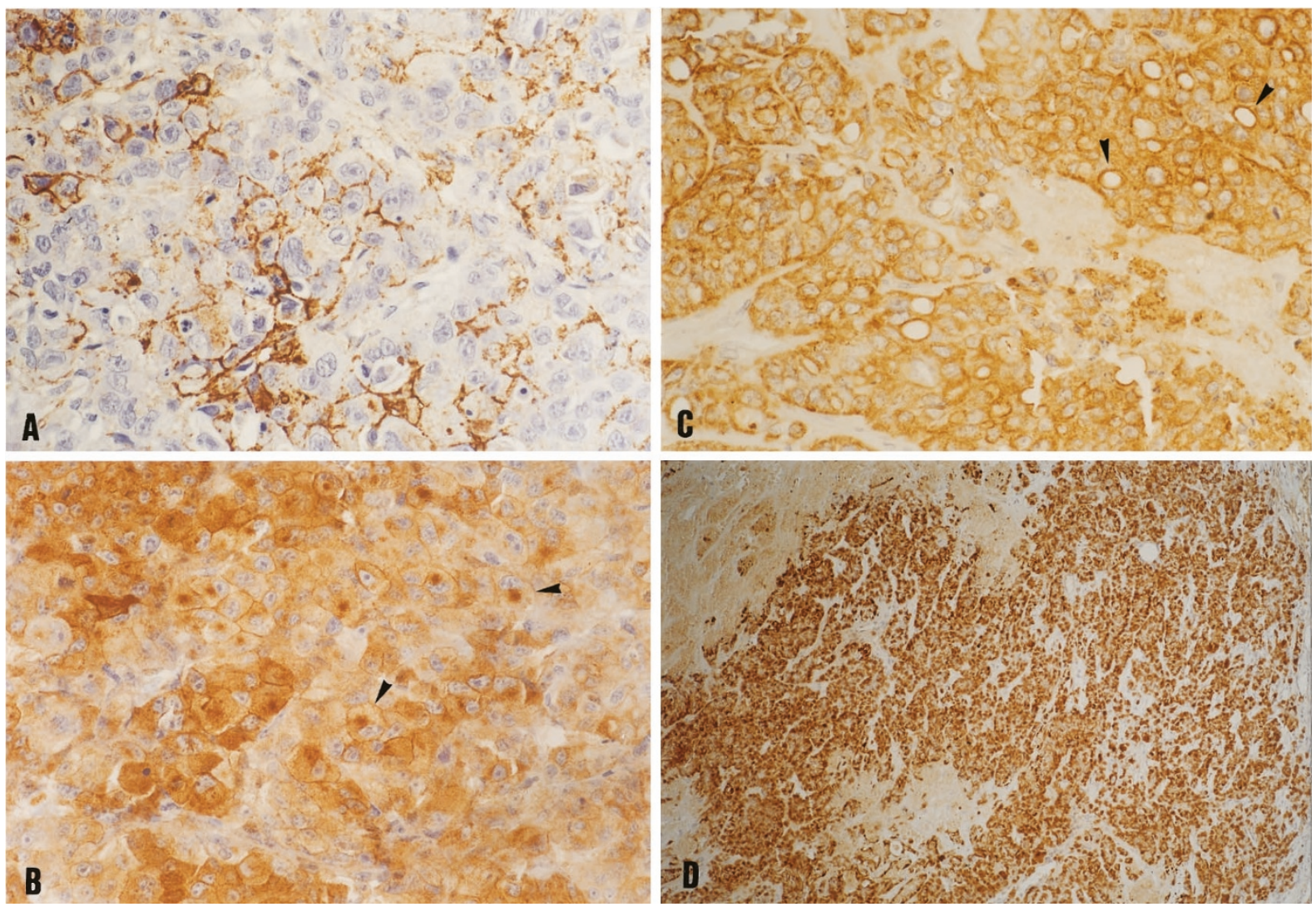

\section{Figure 6.}

$A$, Immunohistological localization of PA2.26 in a lymph node metastasis section. $B$ to $D$, Immunohistological localization of $\mathrm{E}$-cadherin $(B)$, K8 $(C)$, and vimentin $(D)$, in tumors induced by 3D2.26-11 cells. Arrowheads in panel $B$ point to cells with large juxta-nuclear cytoplasmic aggregates of E-cadherin. Note that K8 staining in panel $C$ is more intense at the cell periphery and surrounding cavities (arrowheads).

expression of PA2.26 antigen concomitantly with an epithelial-fibroblastoid transition triggered by other genetic/epigenetic changes.

In the experimental conditions used in this work, 3D2.26 cells were tumorigenic upon injection in nude mice, whereas MCA3D and control transfectants were not (Table 1). The metastatic potential of 3D2.26 clones was assessed by observing infiltration of tumor cells into lymphatic vessels in most of the tumors (Fig. 5 ) and by the presence of regional lymph node metastasis in at least $50 \%$ of the lesions (Figs. 4 and 5; Table 1). Tumors exhibited an undifferentiated phenotype, although some areas showed a glandular differentiation pattern resembling malignant mesotheliomas, as demonstrated at the ultrastructural level by the presence of numerous wavy-appearing surface microvilli (Dardick et al, 1987; Oury et al, 1998). Interestingly, PA2.26 antigen was concentrated at these microvillous surfaces (Fig. 4). This finding suggests that PA2.26 protein is an important component of microvilli, in agreement with our previous results showing PA2.26 localized at microvillous extensions in normal adult tissues (Scholl et al, 1999), and that PA2.26 could be involved in the formation and stabilization of these structures.

The molecular and biochemical characterization of PA2.26 antigen, and of its homologues described by other laboratories in different species (see introduc- tory paragraphs), revealed that they are small mucintype transmembrane sialoglycoproteins located in vivo at the apical surface of epithelial, endothelial, and mesothelial cells (Scholl et al, 1999). Mucin-type membrane glycoproteins have been shown to exhibit both anti-adhesive and cell-cell adhesive properties (Baumhueter et al, 1994; Gendler and Spicer, 1995; Nath et al, 1999). The anti-adhesive function of large transmembrane mucins, such as episialin (MUC1), epiglycanin, and sialomucin, is attributable to their masking cell adhesion receptors because of their large extended ectodomains and to the repulsive forces provided by sialic acid (Kemperman et al, 1994; Komatsu et al, 1997; Wesseling et al, 1996). Nevertheless, the relatively small size estimated for the extracellular domain of PA2.26 antigen (Scholl et al, 1999) makes it unlikely that steric hindrance is the only cause of the profound phenotypic effects induced by PA2.26 in keratinocytes.

PA2.26 antigen appears to associate with actin filaments through binding to either ezrin or moesin. ERM proteins play structural and regulatory roles in the formation of cell-surface extensions by linking actin filaments to transmembrane proteins (Mangeat et al, 1999) and might be involved in the regulation of cell-cell and cell-matrix adhesion (Hiscox and Jiang, 1999). Although the mechanism for the PA2.26induced phenotypic conversion of MCA3D keratino- 
cytes is still not completely understood, we suggest that these changes might be mediated by the interaction of the cytoplasmic domain of PA2.26 with the cytoskeleton. Nevertheless, the possibility of interactions between the PA2.26 ectodomain and the extracellular matrix, cell adhesion, or another type of receptor, as occurs with other transmembrane glycoproteins (Baumhueter et al, 1994; Naor et al, 1997), remains to be investigated.

Expression of PA2.26 in MCA3D cells results in formation of actin-rich filopodia, lamellipodia, and ruffles, recruitment of ezrin to plasma membrane extensions (Scholl et al, 1999), and loss of actin cortical bundles and stress fibers (Fig. 1). Interactions between epithelial cells are stabilized by linkage of cadherin-catenin complexes to the cortical actin network in adherens junctions (Ozawa and Kemler, 1992), and transient or permanent inactivation of adherens junction complexes is required for cell migration/ invasion (Birchmeier and Behrens, 1994; Takeichi, 1993). The establishment of a cortical actin filament network is necessary for localization of cadherincatenin complexes at adherens junctions in epithelial cells. Thus, disturbances of the cortical actin network result in destabilization of adherens junctions and in epithelial cells with E-cadherin found in intracellular vesicles and not at the cell surface (Quinlan and Hyatt, 1999), a phenomenon that contributes to tumor progression (Fischer and Quinlan, 1998). Therefore, loss of cortical actin filaments might account for destabilization of adherens junctions mediated by $\mathrm{E}$ - and P-cadherin in 3D2.26 transfectants. The fact that 3D2.26 cells expressed cadherin transcripts and that only polypeptides smaller than the mature form could be detected for E-cadherin indicates at least that E-cadherin protein synthesized in 3D2.26 cells is unstable and degraded. E-cadherin fragments of similar molecular masses as those seen in 3D2.26 cells have been found to be derived by proteolysis (Shirayoshi et al, 1986). On the other hand, Lozano and Cano (1998) have shown that when E-cadherin is ectopically expressed in spindle carcinoma cells, which lack a cortical actin network, the protein is unstable and associates weakly with the cytoskeleton, despite forming cadherin/catenin complexes similar to those of epithelial cells. The finding that no polypeptides derived from $\mathrm{P}$-cadherin were observed in 3D2.26 cells might indicate that $\mathrm{P}$-cadherin is more unstable than E-cadherin or, alternatively, the existence of a posttranscriptional mechanism for regulation of P-cadherin expression.

Recent studies have emphasized the importance of connections between intermediate filaments and microfilaments and connections with the cell surface for maintaining cellular integrity and properties (Chou et al, 1997). Cytoskeletal organization and cell shape are closely related, and mechanical tension generated within the cytoskeleton can regulate cell shape and cause cells to switch between different genetic programs (Chicurel et al, 1998). For example, in a mammary epithelial wound-healing cell migration model, induction of vimentin expression occurs exclusively at the wound's edge in cells whose actin filaments were reorganized to form lamellipodial extensions (Gilles et al, 1999). Interestingly, we have observed loss of cortical actin and stress fibers, together with downregulation of basal keratins and induction of vimentin and $\mathrm{K} 8$, in transformed keratinocytes treated with transforming growth factor- $\beta_{1} \quad\left(\mathrm{TGF}-\beta_{1}\right)$. These changes were associated with the development of poorly differentiated carcinomas and the acquisition of metastatic abilities (Caulín et al, 1995; Frontelo et al, 1998). These and other studies have shown that the expression of $\mathrm{K} 8$ and vimentin, linked to downregulation of basal keratins, is associated with malignant progression of squamous cell carcinomas (Caulín et al, 1993a, 1993b; Larcher et al, 1992; Markey et al, 1991), and several reports have related coexpression of simple epithelial keratins and vimentin to increased cell migration/invasion (Chu et al, 1996; Hendrix et al, 1997; Schaafsma et al, 1993). Thus, PA2.26-induced reorganization of the actin cytoskeleton could lead to up-regulation of keratin $\mathrm{K} 8$ and vimentin and repression of basal keratin K14 expression. Although the molecular mechanism for these regulatory changes has not been identified, it is likely to involve AP-1 and ETS transcription factors, which have been implicated in the anomalous expression of simple epithelial keratins in carcinomas and of vimentin in migrating epithelial cells (Gilles et al, 1999; Oshima et al, 1996).

Although a precise physiological role for PA2.26 antigen is still difficult to assign, the results presented here demonstrate that forced expression of PA2.26 in premalignant keratinocytes can induce a fully transformed and metastatic phenotype. This finding, together with our previous observation showing the induction of PA2.26 during mouse epidermal carcinogenesis (Gandarillas et al, 1997), suggests that PA2.26 antigen might be functionally involved in malignancy.

\section{Materials and Methods}

\section{Cell Culture Conditions and DNA Transfection}

The keratinocyte cell lines used in this study, MCA3D, $\mathrm{PDV}$, and $\mathrm{HaCa} 4$, have been described in detail in different reports (Caulín et al, 1995; Gandarillas et al, 1997; Kulesz-Martin et al, 1983; Navarro et al, 1991; Quintanilla et al, 1991). Cells were grown in Ham's F-12 medium supplemented with amino acids, $10 \%$ fetal calf serum, and antibiotics $(2.5 \mu \mathrm{g} / \mathrm{ml}$ amphotericin B, $100 \mu \mathrm{g} / \mathrm{ml}$ ampicillin, and $32 \mu \mathrm{g} / \mathrm{ml}$ gentamicin; Sigma-Aldrich, Madrid, Spain).

Transfections of MCA3D cells with the mouse PA2.26 cDNA cloned into the pcDNA3 expression vector, or with the pcDNA3 vector alone, have been described elsewhere (Scholl et al, 1999). Transfected cells were selected in G418 $(0.4 \mathrm{mg} / \mathrm{ml})$ for 3-4 weeks, and clones were isolated by cloning rings.

\section{RT-PCR, Northern, and Western Blot Analysis}

Poly $(A)^{+}$-enriched mRNA was isolated from the different cell lines using the Mini RiboSep isolation kit (Becton Dickinson Labware, Bedford, Massachusetts). RT-PCR was carried out with specific primers. The following 
sense/antisense primers were used: 5'-CGTGATGAAGGTCTCAGCC-3'/5'-ATGGGGGCATTCATTCAC-3', for E-cadherin; 5'-GTAATGCCTGGCACTTCTG-3'/5'CGCCTTGGTTGGTCTCTG-3', for P-cadherin; and 5'-TGAAGGTCGGTGTGAACGGATTTGGC-3'/5'-CATGTAGGCCATGAGGTCCACCAC-3', for glyceraldehyde-3phosphate dehydrogenase (GPDH). Primers for PA2.26 and RT-PCR conditions have been described (Scholl et al, 1999).

Northern blot hybridization experiments were performed with total RNA isolated by the guanidiniumthiocyanate procedure. Denatured RNAs $(20 \mu \mathrm{g})$ were loaded on $1 \%$ agarose-formaldehyde gels and subjected to electrophoresis. mRNAs were transferred to nylon membranes (Zeta Probe, BioRad, Madrid, Spain). Probes used for detection of K8, K14, and vimentin mRNAs have been described previously (Caulín et al, 1995; Frontelo et al, 1998).

For detection of cadherins and $\beta$-catenin in Western blots, cells were lysed in a detergent buffer containing $10 \mathrm{~mm} \mathrm{CaCl}_{2}$ to preserve cadherin integrity as described (Navarro et al, 1991). Samples containing identical amounts of total proteins were run on $10 \%$ SDS-polyacrylamide gel electrophoresis and transferred to Immobilon P membranes (Millipore Corporation, Bedford, Massachusetts). Filters were immunoblotted with mAbs ECCD-2, PCD-1 (Zymed Laboratories Inc., San Francisco, California), and C14 (Transduction Laboratories, Lexington, Kentucky), specific for E-cadherin, P-cadherin, and $\beta$-catenin, respectively. ECCD-2 was a generous gift of Dr. Masatoshi Takeichi (Kyoto University, Kyoto, Japan). Appropriate secondary antibodies coupled to horseradish peroxidase (Amersham Corporation, Arlington Heights, Florida) were used. The peroxidase activity was developed by using an enhanced chemiluminescence kit (ECL, Amersham Corporation).

\section{Immunofluorescence, Histological, and Electron Microscopy Analysis}

Immunofluorescent detection of E- and P-cadherin was performed in confluent cells grown on glass coverslips, washed in buffer containing $2.5 \mathrm{mM} \mathrm{CaCl}_{2}$, fixed in $3.7 \%$ formaldehyde in PBS for 30 minutes, and permeabilized in $0.05 \%$ Triton $\mathrm{X}-100$ for 10 minutes, using ECCD-2 (1:100) and PCD-1 (1:100) mAbs, as previously described (Navarro et al, 1991). Detections of intermediate filament proteins were carried out in cells fixed and permeabilized in cold-methanol, at $-20^{\circ} \mathrm{C}$ for 5 minutes, with the following monoclonal antibodies: TROMA-1 for detection of K8, LL001 (a kind gift of Dr. E. Birgitte Lane, University of Dundee, Dundee, United Kingdom) for detection of K14, and VIM3B4 (Progen Immuno-Diagnostika, Heidelberg, Germany) for detection of vimentin (Caulín et al, 1993a; Frontelo et al, 1998), all at 1:100 dilution. FITCor rhodamine-conjugated secondary antibodies were purchased from Jackson Immunoresearch (West Grove, Pennsylvania). For actin filaments staining, phalloidin coupled with rhodamine compound was used. Coverslips were mounted in Mowiol and exam- ined with a fluorescence microscope (Axiophot; Carl Zeiss, Oberkochen, Germany).

Tumors were fixed in formalin and embedded in paraffin. Immunostainings were performed in deparaffinized sections, after heat-induced antigen retrieval, by the Envision plus peroxidase method (Dako A/S, Glostrup, Denmark). C36 (1:200) and C56 (1:100) mAbs (Transduction Laboratories) were used as primary antibodies for detection of E- and P-cadherin, respectively; TROMA-1 (1:100) was used for detection of K8; and VIM3B4 (1:100) was used for detection of vimentin. The reaction product was developed with diaminobenzidine tetrahydrochloride and $\mathrm{H}_{2} \mathrm{O}_{2}$. The sections were dehydrated in graded ethanols, cleared in xylene, and mounted in Permount after counterstain with hematoxylin and eosin (H\&E).

Electron microscopy analysis was performed on tissue fixed in 2.5\%-buffered glutaraldehyde and postfixed in $\mathrm{OsO}_{4}$. Specimens were embedded in Epon and mounted on 300-mesh copper grids. Ultrathin sections $(0.1-0.2 \mu \mathrm{m})$ were stained with uranyl acetate and lead citrate and examined in a Philips CM10 electron microscope. Semithin sections $(2 \mu \mathrm{m})$ were cut with glass knives and stained with toluidine blue.

\section{Tumorigenicity Assays}

Cells were collected, washed in PBS, and intradermally injected into the two flanks (Experiment 1), or in a single injection in the back close to the neck (Experiment 2), of 8-week-old Swiss athymic nude mice (Animal Production Area, CRIFFA, Barcelona, Spain). Approximately $3 \times 10^{6}$ viable cells were used for each injection. Animals were observed for tumor formation and the size of tumors calculated from caliper measurements of two orthogonal diameters at different times. At the indicated postinjection times, animals were anesthetized and killed. In Experiment 2, lymphadenopathies were observed by gross examination of the tumors, and the presence of regional lymph node metastasis was assessed by histological analysis. Histological typing of tumors and lymph node metastases was performed in $10 \%$ formalin-fixed and paraffin-embedded sections by staining with H\&E.

\section{Acknowledgements}

We thank Drs. Masatoshi Takeichi and E. Birgitte Lane for their kind gifts of ECCD-2 and LLOO1 monoclonal antibodies, respectively, and Dr. Amparo Cano for her help in cadherin studies. We also thank Ms. Petra Rubio and Ms. Raquel Marcos for histological technical assistance.

\section{References}

Baumhueter S, Dybdal N, Kyle C, and Lasky LA (1994). Global vascular expression of murine CD34, a sialomucin-like endothelial ligand for L-selectin. Blood 84:2554-2565.

Birchmeier W and Behrens J (1994). Cadherin expression in carcinomas: Role in the formation of cell junctions and the prevention of invasiveness. Biochim Biophys Acta 1198:1126. 
Breiteneder-Geleff S, Matsui K, Soleiman A, Meraner P, Poczewski H, Kalt R, Schaffner G, and Kerjaschki D (1997). Podoplanin, novel 43-kd membrane protein of glomerular epithelial cells, is down-regulated in puromycin nephrosis. Am J Pathol 151:1141-1152.

Burns PA, Kemp CJ, Gannon JV, Lane DP, Bremner R, and Balmain A (1991). Loss of heterozygosity and mutational alterations of the p53 gene in skin tumours of interspecific hybrid mice. Oncogene 6:2363-2369.

Caulín C, Bauluz C, Gandarillas A, Cano A, and Quintanilla M. (1993a). Changes in keratin expression during malignant progression of transformed mouse epidermal keratinocytes. Exp Cell Res 204:11-21.

Caulín C, Gandarillas A, Scholl FG, and Quintanilla M. (1993b). Expression of intermediate filament proteins by normal and transformed mouse epidermal keratinocytes in culture. Int J Oncol 2:373-379.

Caulín C, Scholl FG, Frontelo P, Gamallo C, and Quintanilla M (1995). Chronic exposure of cultured transformed mouse epidermal cells to transforming growth factor- $\beta_{1}$ induces an epithelial-mesenchymal transdifferentiation and a spindle tumoral phenotype. Cell Growth Differ 6:1027-1035.

Chicurel ME, Chen CS, and Ingber DE (1998). Cellular control lies in the balance of forces. Curr Opin Cell Biol 10:232-239.

Chou YH, Skalli O, and Goldman RD (1997). Intermediate filaments and cytoplasmic networking: new connections and more functions. Curr Op Cell Biol 9:49-53.

Chu YW, Seftor EA, Romer LH, and Hendrix MJ (1996). Experimental coexpression of vimentin and keratin intermediate filaments in human melanoma cells augments motility. Am J Pathol 148:63-69.

Dardick I, Jabi M, McCaughey WT, Deodhare S, van Nostrand AW, and Srigley JR (1987). Diffuse epithelial mesothelioma: a review of the ultrastructural spectrum. UItrastruct Pathol 11:503-533.

Farr AG, Berry ML, Kim A, Nelson AJ, Welch MP, and Aruffo $A$ (1992). Characterization and cloning of a novel glycoprotein expressed by stromal cells in T-dependent areas of peripheral lymphoid tissues. J Exp Med 176:1477-1482.

Fischer RS and Quinlan MP (1998). Identification of a novel mechanism of regulation of the adherens junction by $E 1 A$, Rac1 and cortical actin filaments that contributes to tumor progression. Cell Growth Differ 9:905-918.

Frontelo P, González-Garrigues M, Vilaró S, Gamallo C, Fabra A, and Quintanilla M (1998). Transforming growth factor- $\beta_{1}$ induces squamous carcinoma cell variants with increased metastatic abilities and a disorganized cytoskeleton. Exp Cell Res 244:420-432.

Gandarillas A, Scholl FG, Benito N, Gamallo C, and Quintanilla M (1997). Induction of PA2.26, a cell-surface antigen expressed by active fibroblasts, in mouse epidermal keratinocytes during carcinogenesis. Mol Carcinog 20:10-18.

Gendler SJ and Spicer AP (1995). Epithelial mucin genes. Annu Rev Physiol 57:607-634.

Gilles C, Polette M, Zahm JM, Tournier JM, Volders L, Foidart JM, and Birembaut $P$ (1999). Vimentin contributes to human mammary epithelial cell migration. J Cell Sci 112:4615-4625.
Hendrix MJ, Seftor EA, Seftor RE, and Trevor KT (1997). Experimental co-expression of vimentin and keratin intermediate filaments in human breast cancer cells results in phenotypic interconversion and increased invasive behavior. Am J Pathol 150:483-495.

Hiscox S and Jiang WG (1999). Ezrin regulates cell-cell and cell-matrix adhesion, a possible role with $\mathrm{E}$-cadherin/ $\beta$ catenin. J Cell Sci 112:3081-3090.

Kemperman H, Wijnands $Y$, Wesseling J, Niessen CM, Sonnenberg $A$, and Roos $E$ (1994). The mucin epiglycanin on TA3/Ha carcinoma cells prevents alpha 6 beta 4-mediated adhesion to laminin and kalinin and E-cadherin-mediated cell-cell interaction. J Cell Biol 127:2071-2080.

Komatsu M, Carraway CA, Fregien NL, and Carraway KL (1997). Reversible disruption of cell-matrix and cell-cell interactions by overexpression of sialomucin complex. J Biol Chem 272:33245-33254.

Kulesz-Martin M, Kilkenny AE, Holbrook KA, Digernes V, and Yuspa SH (1983). Properties of carcinogen altered mouse epidermal cells resistant to calcium-induced terminal differentiation. Carcinogenesis 4:1367-1377.

Larcher F, Bauluz C, Díaz-Guerra M, Quintanilla M, Conti CJ, Ballestin C, and Jorcano JL (1992). Aberrant expression of the simple epithelial type II keratin 8 by mouse skin carcinomas but not papillomas. Mol Carcinog 6:112-121.

Linardopoulos S, Street AJ, Quelle DE, Parry D, Peters G, Sherr CJ, and Balmain A (1995). Deletion and altered regulation of $\mathrm{p} 16^{\mathrm{INK} 4 \mathrm{a}}$ and $\mathrm{p} 15^{\mathrm{INK} 4 \mathrm{~b}}$ in undifferentiated mouse skin tumors. Cancer Res 55:5168-5172.

Lozano E and Cano A (1998). Induction of mutual stabilization and retardation of tumor growth by coexpression of plakoglobin and E-cadherin in mouse skin spindle carcinoma cells. Mol Carcinog 21:273-287.

Markey AC, Lane EB, Churchill LJ, MacDonald DM, and Leigh IM (1991). Expression of simple epithelial keratins 8 and 18 in epidermal neoplasia. J Invest Dermatol 97:763770 .

Mangeat P, Roy C, and Martin M (1999). ERM proteins in cell adhesion and membrane dynamics. Trends Cell Biol 9:187192.

Naor D, Sionov RV, and Ish-Salom D (1997). CD44: Structure, function and association with the malignant process. Adv Cancer Res 71:241-319.

Nath D, Hartnell A, Happerfield L, Miles DW, Burchell J, Taylor-Papadimitriou J, and Crocker PR (1999). Macrophage-tumor cell interactions: Identification of MUC1 on breast cancer cells as a potential counter-receptor for the macrophage-restricted receptor, sialoadhesin. Immunology 98:213-219.

Navarro P, Gómez M, Pizarro A, Gamallo C, Quintanilla M, and Cano A (1991). A role for the E-cadherin cell-cell adhesion molecule during tumor progression of mouse epidermal carcinogenesis. J Cell Biol 115:517-533.

Nose K, Saito H, and Kuroki T (1990). Isolation of a gene sequence induced later by tumor-promoting 12-Otetradecanoylphorbol-13-acetate in mouse osteoblastic cells (MC3T3-E1) and expressed constitutively in ras-transformed cells. Cell Growth Differ 1:511-518.

Oshima RG, Baribault H, and Caulín C (1996). Oncogenic regulation and function of keratin 8 and 18. Cancer Metastasis Rev 15:445-471. 
Oury TD, Hammar SP, and Roggli VL (1998). Ultrastructural features of diffuse malignant mesotheliomas. Hum Pathol 29:1382-1392.

Ozawa M and Kemler R (1992). Molecular organization of the uvomorulin-catenin complex. J Cell Biol 116:989-996.

Quinlan MP and Hyatt J (1999). Establishment of the circumferential actin filament network is a prerequisite for localization of the cadherin-catenin complex in epithelial cells. Cell Growth Differ 10:839-854.

Quintanilla M, Haddow S, Jonas D, Jaffe D, Bowden GT, and Balmain A (1991). Comparison of ras activation during epidermal carcinogenesis in vitro and in vivo. Carcinogenesis 12:1875-1881.

Rishi AK, Joyce-Brady M, Fisher J, Dobbs LG, Floros J, Van der Spek J, Brody JS, and Williams MC (1995). Cloning, characterization, and development expression of a rat lung alveolar type I cell gene in embryonic endodermal and neural derivatives. Dev Biol 167:294-306.

Schaafsma HE, van der Velden LA, Manni JJ, Peters H, Link M, Rutter DJ, and Ramaekers FC (1993). Increased expression of cytokeratins 8:18 and vimentin in the invasion front of mucosal squamous cell carcinoma. J Pathol 170:77-86.

Scholl FG, Gamallo C, Vilaró S, and Quintanilla M (1999). Identification of PA2.26 antigen as a novel cell-surface mucin-type glycoprotein that induces plasma membrane extensions and increased motility in keratinocytes. J Cell Sci 112:4601-4613.

Shirayoshi Y, Nose A, Iwasaki K, and Takeichi M (1986). $\mathrm{N}$-linked oligosaccharides are not involved in the function of a cell-cell binding glycoprotein E-cadherin. Cell Struct Funct 11:245-252.
Strickland JE, Greenhalgh DA, Koceva-Chyla A, Hennings H, Restrepo C, Balaschak M, and Yuspa SH (1988). Development of murine epidermal cell lines which contain an activated ras $^{\mathrm{Ha}}$ oncogene and form papillomas in skin grafts on athymic nude mouse hosts. Cancer Res 48:165-169.

Takeichi M (1993). Cadherins in cancer: Implications for invasion and metastasis. Curr Opin Cell Biol 5:806-811.

Wesseling J, van der Valk SW, and Hilkens J (1996). A mechanism for inhibition of E-cadherin-mediated cell-cell adhesion by the membrane-associated mucin episialin/ MUC1. Mol Biol Cell 7:565-577.

Wetterwald A, Hoffstetter W, Cecchini MG, Lanske B, Wagner C, Fleisch H, and Atkinson M (1996). Characterization and cloning of the E11 antigen, a marker expressed by rat osteoblasts and osteocytes. Bone 18:25-132.

Zimmer G, Klenk HD, and Herrler G (1995). Identification of a 40-kDa cell surface sialoglycoprotein with the characteristics of a major influenza $\mathrm{C}$ virus receptor in a Madin-Darby canine kidney cell line. J Biol Chem 270:17815-17822.

Zimmer G, Lottspeich F, Maisner A, Klenk HD, and Herrler G (1997). Molecular characterization of gp40, a mucin-type glycoprotein from the apical plasma membrane of MadinDarby canine kidney cells (type I). Biochem J 326:99-108.

Zimmer G, Oeffner F, von Messling V, Tschernig T, Grönes HJ, Klenk HD, and Herrler G (1999). Cloning and characterization of human gp36, a human mucin-type glycoprotein preferentially expressed in vascular endothelium. Biochem $\mathrm{J}$ 341:277-284. 